\section{Two-Dimensional Displacement and Alignment Sensor Based on Reflection Coefficients of Open Microstrip Lines loaded with Split Ring Resonators}

Ali K. Horestani, Jordi Naqui, Derek Abbott, Christophe Fumeaux, and Ferran Martín

This letter proposes a two-dimensional displacement and alignment sensor based on two open-ended transmission lines, each loaded with a split ring resonator (SRR). In this arrangement, the depth of resonanceinduced notches in the reflection coefficients can be used to sense a displacement of the loading SRRs in two orthogonal directions. Since the operation principle of the sensor is based on the symmetry properties of SRR-loaded transmission lines, the proposed sensor benefits from immunity to variations in ambient conditions. More importantly, it is shown that in contrast to previously published metamaterial-inspired twodimensional displacement and alignment sensors, the proposed sensor can be operated at a single fixed frequency. The concept and simulation results are validated through measurement.

Introduction: In recent years, the application of metamaterial-inspired resonators such as split ring resonators (SRRs) to sensors has attracted an increasing interest [1, 2, 3, 4]. This has arisen because of the subwavelength dimensions, high quality factor resonance and sensitivity of the resonance characteristics of these elements to their physical dimensions and constituent materials. Various types of displacement and rotation sensors based on variation of resonance frequency, quality factor and notch depth in the transmission spectrum of SRR-loaded transmission lines (TLs) have been proposed in the literature $[1,5,6,7,8]$. The concept was also extended for the design of two-dimensional displacement sensors [9, 10].

This letter proposes a two-dimensional displacement and alignment sensor based on the variation in the depth of resonant notch in the reflection coefficients of two SRR-loaded microstrip lines. Since both SRRs and microstrip lines are inherently compact structures, the proposed sensor benefits form a small size. Furthermore, since the operation principle of the sensor is based on the symmetry properties of the SRRs, the sensor is robust to ambient conditions such as changes in temperature. It is also shown that because the excitation from microstrip lines imposes an almost constant loading effect on the SRRs, displacement is only manifested in the depth of notch, leaving the resonance frequency intact. This is an important feature because it enables the sensor to work at a fixed frequency. This sensing mode allows to operate based on two reflection coefficients rather than using the transmission coefficient of the structure, where at least two fixed frequencies are required for the operation of two-dimensional displacement sensors $[9,10]$.

Two-Dimensional Displacement and Alignment Sensor: Illustrations of the top and side views of the proposed sensor are depicted in Figs. 1(a) and (b). The structure is composed of two substrates. On the bottom substrate, two open-ended microstrip lines along $x$ and $y$ axis are excited at Port 2 and Port 1, respectively. Each TL is loaded with an SRR, which is patterned on the top substrate. As shown in the side view of the structure in Fig. 1(b), the second substrate is separated from the first substrate by an air gap, and can be moved in $x$ and $y$ directions. At the initial position, the symmetry plane of each of the SRRs is aligned with the symmetry plane of the corresponding TLs. Thus, due to the symmetry of the structure the SRRs are not excited by the TLs, and full reflection is observed at both ports. However, if the alignment is broken by a displacement in $x$ and/or $y$ direction(s), the SRR(s) will be excited and notch(es) will appear in the corresponding reflection coefficient(s). Further displacement in $x$ and/or $y$ directions results in a stronger coupling between the TLs and the SRRs, in turn resulting in deeper notch(es) in the respective reflection coefficient(s) $\left(\left|S_{11}\right|\right.$ and $\left.\left|S_{22}\right|\right)$ at the resonant frequency of the SRRs. Note that displacement in $x$ direction have no effect on the depth of notch in $\left|S_{22}\right|$ and vice versa. Thus, misalignment in $x$ and $y$ directions can be independently sensed from the depth of notches in $\left|S_{11}\right|$ and $\left|S_{22}\right|$, respectively. One advantage of this method is that both SRRs can be designed to operate at the same resonance frequency. This is in contrast to the previously proposed two-dimensional displacement sensors based on transmission characteristics of a through TL, where SRRs needed to have distinct resonance frequencies $[9,10]$.

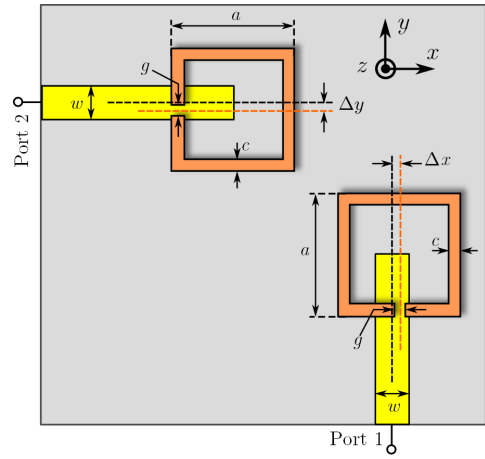

(a)

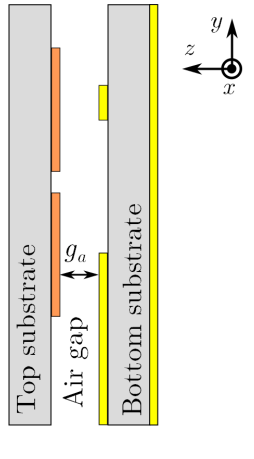

(b)
Fig. 1 (a) Top view and (b) side view (b) of the proposed two-dimensional displacement and alignment sensor.

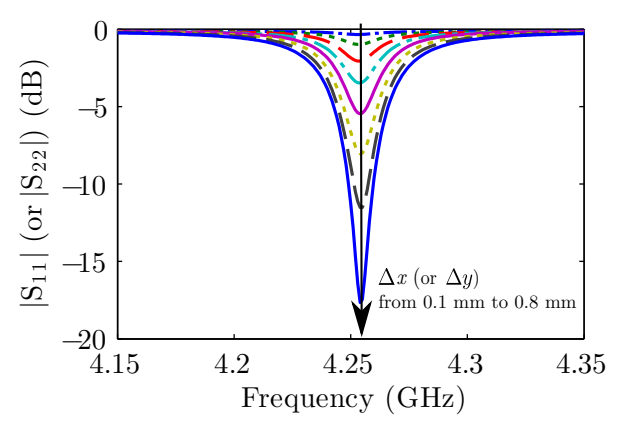

Fig. 2 Simulated $\left|S_{11}\right|$ (or $\left|S_{22}\right|$ ) of the structure for different values of displacement $\Delta x$ (or $\Delta y$ ) from $0.1 \mathrm{~mm}$ to $0.8 \mathrm{~mm}$ in steps of $0.1 \mathrm{~mm}$. Note that due to the symmetry of the structure, behavior of $\left|S_{22}\right|$ with respect to $\Delta y$ is identical to that of $\left|S_{11}\right|$ with respect to $\Delta x$.

For electromagnetic simulations, two $0.81 \mathrm{~mm}$ thick Rogers $\mathrm{RO4003}$ substrates $\left(\epsilon_{r}=3.38\right.$ and $\left.\tan \delta=0.0022\right)$ with $35 \mu \mathrm{m}$ thick copper metalization are used. The dimensions of the $50 \Omega$ microstrip lines and the square SRRs are as follows: $w=1.84 \mathrm{~mm}, a=7 \mathrm{~mm}, g=0.5 \mathrm{~mm}$, $c=0.5 \mathrm{~mm}$, and $g_{a}=0.76 \mathrm{~mm}$. Figure 2 shows the simulated reflection coefficient of the proposed sensor at Port 1 for different values of $\Delta x$ from $0.1 \mathrm{~mm}$ to $0.8 \mathrm{~mm}$ in steps of $0.1 \mathrm{~mm}$. Since both SRRs have identical dimensions, the simulated $\left|S_{22}\right|$ versus $\Delta y$ has an identical behavior. The figure clearly shows the variation of notch depth in $\left|S_{11}\right|$ (or $\left|S_{22}\right|$ ) with displacement in $x$ (or $y$ ) direction. An important feature of the proposed sensor is that since the excitation from the TLs imposes an almost constant-predominantly electrical-loading effect on the SRRs, a displacement only affects the depth of notch, without altering the resonance frequency. This is in contrast to the previously proposed displacement sensors in which, due to cross-polarized coupling and loading effects, a displacement affected both depth of notch and resonance frequency $[5,6,8,11]$.

In order to investigate the mutual effect of displacement in $x$ and $y$ directions on the functionality of the sensor, Fig. 3 shows the simulated $\left|S_{11}\right|$ versus displacement in $x$ direction, at a fixed frequency $f=$ $4.253 \mathrm{GHz}$, while displacement in $y$ direction is changed from $0 \mathrm{~mm}$ to $0.8 \mathrm{~mm}$ in steps of $0.2 \mathrm{~mm}$. Obviously, due to the symmetry of the structure, an identical behavior is expected for the effect of $\Delta x$ on $\left|S_{22}\right|$ versus $\Delta y$. The figure shows that sensing relatively large values of $\Delta x$ is slightly affected by displacement in $y$ direction, however, $\Delta y$ has almost no effect on sensing smaller values of $\Delta x(\leqslant 0.4 \mathrm{~mm})$. This is a crucial feature of the proposed structure for application as a two-dimensional alignment sensor.

Experimental Results: In order to validate the concept and the simulation results, a prototype of the sensor has been fabricated and measured. Figure 4 depicts the photograph of the fabricated prototype, which includes two microstrip lines fabricated on the first substrate, while the SRRs are etched on the movable top substrate. As in the simulations, Rogers RO4003 material is used for both substrates. The dimensions of the fabricated microstrip lines and SRRs correspond to the dimensions of the simulated structure. 


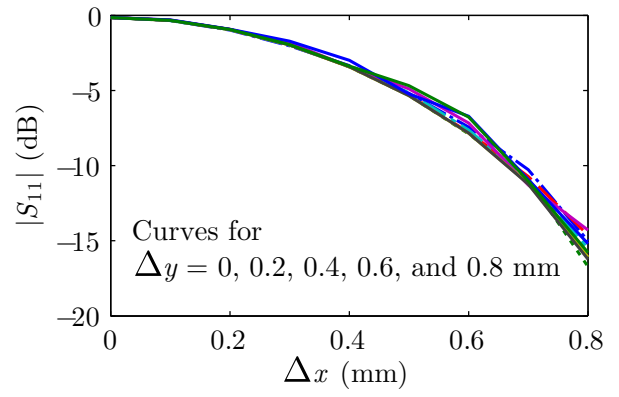

Fig. 3 Simulated depth of notch versus $\Delta x$, at the fix frequency $f=$ $4.253 \mathrm{GHz}$, for different values of $\Delta y$ from $0 \mathrm{~mm}$ to $0.8 \mathrm{~mm}$ in steps of $0.2 \mathrm{~mm}$. Due to the symmetry of the proposed sensor, an identical plot also describes dependency to displacement in x direction for $\left|S_{22}\right|$ versus $\Delta y$.

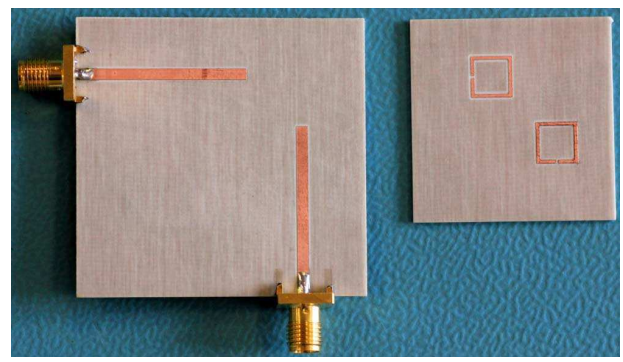

Fig. 4 Photograph of a fabricated prototype of the sensor. The two microstrip lines are printed on the fixed substrate (left), and the SRRs are fabricated on the movable substrate (right). Rogers RO4003 material is used for both substrates.

The experimental setup is composed of three pairs of micrometer actuators allowing to move the top substrate in three directions. The first pair of micrometer actuators are used to precisely adjust the air gap between the two substrates to $g_{a}=0.76 \mathrm{~mm}$. The other two pairs of micrometer actuators are used to control displacements in $x$ and $y$ directions from $0 \mathrm{~mm}$ to $0.8 \mathrm{~mm}$ in steps of $0.1 \mathrm{~mm}$, while the reflection coefficients at Port 1 and Port 2 are measured. Figure 5 depicts the measured reflection coefficient of the sensor at Port 1 for different values of displacement in $x$ direction. Due to the symmetry of the structure, the reflection coefficient at Port 2 has the same behavior with respect to displacement in $y$ direction. Thus, the experimental results clearly shows that notch depth in the reflection coefficients at Port 1 and Port 2 of the structure can be used to sense a displacement in $x$ and $y$ directions, respectively.

As mentioned earlier, one important advantage of the proposed sensor is that due to almost constant loading effect of the TLs on the SRRs, displacement only affects the dept of notch, and leaves the resonance frequency intact. This is an important feature that enables the proposed sensor to operate at a fixed frequency, bypassing the need for a frequency sweeping source and measurement system. In order to validate this important feature, Fig. 6 depicts the simulated (red dashed lines) and measured (blue solid lines) $\left|S_{11}\right|$ and $\left|S_{22}\right|$ at fixed frequency of $4.253 \mathrm{GHz}$ versus $\Delta x$, while $\Delta y$ is $0 \mathrm{~mm}$. The figure clearly shows that movement in $x$ direction can be sensed from $\left|S_{11}\right|$, while $\left|S_{22}\right|$ remains unaffected and shows the alignment in $y$ direction. The good agreement between the

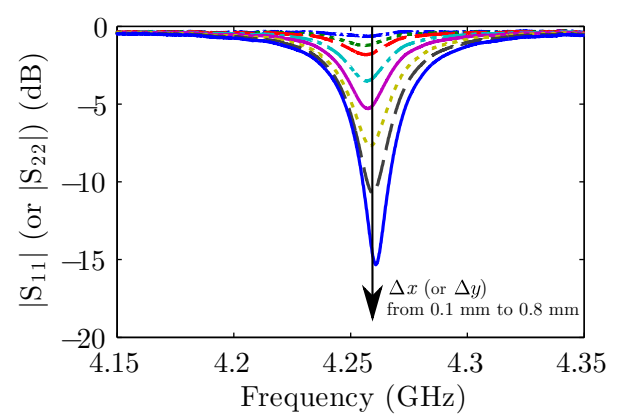

Fig. 5 Measured $\left|S_{11}\right|$ (or $\left|S_{22}\right|$ ) of the structure for different values of displacement $\Delta x$ (or $\Delta y$ ) from $0.1 \mathrm{~mm}$ to $0.8 \mathrm{~mm}$ in steps of $0.1 \mathrm{~mm}$.

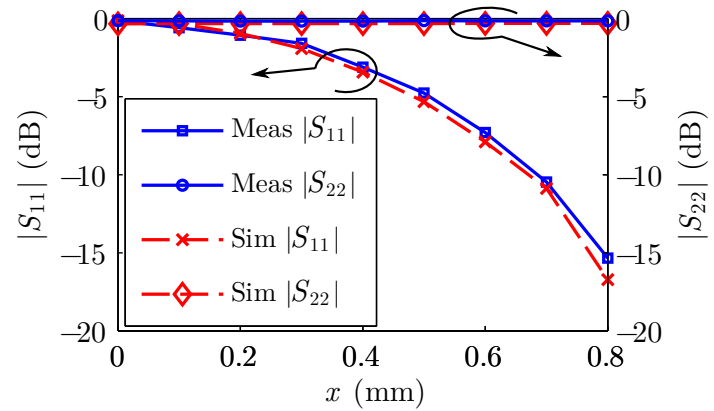

Fig. 6 A comparison between measured and simulated $\left|S_{11}\right|$ and $\left|S_{22}\right|$ at the fixed frequency $f=4.253 \mathrm{GHz}$ versus $\Delta x$, for $\Delta y=0 \mathrm{~mm}$. Due to the symmetry of the proposed sensor, an identical comparison is valid between measured and simulated $\left|S_{11}\right|$ and $\left|S_{22}\right|$ versus $\Delta y$.

curves validates the simulation results, and confirms the operation of the sensor at a fixed frequency.

Conclusion: A two-dimensional displacement and alignment sensor based on the reflection characteristics of two open-ended microstrip lines loaded with two SRRs has been presented. The proposed sensor is compact and its operation is robust to ambient conditions such as changes in the temperature, as it is operated based on break of symmetry and only requires measurement at a single fixed frequency. This is an important feature that bypasses the need for a frequency sweeping measurement system such as a network analyzer. The good agreement between the numerical and experimental results validates the design principle.

Ali K. Horestani, Derek Abbott, Christophe Fumeaux (School of Electrical \& Electronic Engineering, The University of Adelaide, Adelaide, SA 5005, Australia), Jordi Naqui and Ferran Martín (GEMMA/CIMITEC, Departament d'Enginyeria Electronica, Universitat Autonoma de Barcelona, 08193 Bellaterra, Spain)

E-mail: Ali.K.Horestani@ieee.org

\section{References}

1 M. Schueler, C. Mandel, M. Puentes, and R. Jakoby, "Metamaterial inspired microwave sensors," IEEE Microw. Mag., vol. 13, no. 2, pp. 5768, Mar. 2012.

2 W. Withayachumnankul, K. Jaruwongrungsee, C. Fumeaux, and D. Abbott, "Metamaterial-inspired multichannel thin-film sensor," IEEE Sensors J., vol. 12, no. 5, pp. 1455-1458, May 2012.

3 S. A. Taya and M. M. Shabat, "Sensitivity enhancement in optical waveguide sensors using metamaterials," Applied Physics A, vol. 103, no. 3, pp. 611-614, May 2011.

4 A. Ebrahimi, W. Withayachumnankul, S. Al-Sarawi, and D. Abbott, "High-sensitivity metamaterial-inspired sensor for microfluidic dielectric characterization," IEEE Sensors J., vol. 14, no. c, pp. 1-7, 2014.

5 J. Naqui, M. Durán-Sindreu, and F. Martín, "Novel sensors based on the symmetry properties of split ring resonators (SRRs)." Sensors, vol. 11, no. 8, pp. 7545-53, Jul. 2011.

6 A. K. Horestani, C. Fumeaux, S. Al-Sarawi, and D. Abbott, "Displacement sensor based on diamond-shaped tapered split ring resonator,' IEEE Sensors J., vol. 13, no. 4, pp. 1153-1160, Apr. 2013.

7 Z. Shaterian, A. K. Horestani, and C. Fumeaux, "MetamaterialInspired Displacement Sensor with High Dynamic Range," in Proc. 4th International Conference on Metamaterials, Photonic Crystals and Plasmonics, META'13, vol. 1, Sharjah, United Arab Emirates, Mar. 2013, pp. $9-11$.

8 A. K. Horestani, D. Abbott, and C. Fumeaux, "Rotation sensor based on horn-shaped split ring resonator," IEEE Sensors J., vol. 13, no. 8, pp. 3014-3015, Aug. 2013.

9 J. Naqui, M. Durán-Sindreu, and F. Martín, "Alignment and position sensors based on split ring resonators," Sensors, vol. 12, no. 9, pp. $11790-11797$, Jan. 2012.

10 A. K. Horestani, J. Naqui, Z. Shaterian, D. Abbott, C. Fumeaux, and F. Martín, "Two-dimensional alignment and displacement sensor based on movable broadside-coupled split ring resonators," Journal of Sensors and Actuators A: Physical in print, 2014.

11 J. Naqui, M. Duran-Sindreu, and F. Martin, "Modeling split-ring resonator (SRR) and complementary split-ring resonator (CSRR) loaded transmission lines exhibiting cross-polarization effects," IEEE Antennas Wireless Propag. Lett., vol. 12, pp. 178-181, 2013. 